\title{
On a rigidity result for the first conformal eigenvalue of the Laplacian
}

\author{
Romain Petrides
}

\begin{abstract}
Given $(M, g)$ a smooth compact Riemannian manifold without boundary of dimension $n \geq 3$, we consider the first conformal eigenvalue which is by definition the supremum of the first eigenvalue of the Laplacian among all metrics conformal to $g$ of volume 1 . We prove that it is always greater than $n \omega_{n}^{\frac{2}{n}}$, the value it takes in the conformal class of the round sphere, except if $(M, g)$ is conformally diffeomorphic to the standard sphere.
\end{abstract}

Mathematics Subject Classification (2010). Primary 58; Secondary 46, 49.

Keywords. Bounds, conformal, eigenvalue, Laplacian, conformal volume, Riemannian manifold.

Let $(M, g)$ be a smooth compact Riemannian manifold without boundary of dimension $n \geq 3$ and let us define the first conformal eigenvalue of $(M, g)$ by

$$
\Lambda_{1}(M,[g])=\sup _{\tilde{g} \in[g]} \lambda_{1}(M, \tilde{g}) \operatorname{Vol}_{\tilde{g}}(M)^{\frac{2}{n}}
$$

where $\lambda_{1}(M, g)$ is the first nonzero eigenvalue of the Laplacian $\Delta_{g}=-\operatorname{div}_{g}(\nabla)$ and $[g]$ is the conformal class of $g$. In this paper, we aim at proving a rigidity result concerning this first conformal eigenvalue.

The maximisation on conformal classes is natural because the scale invariant quantity supremum is infinite among all metrics [3] (except in dimension 2, [16]), while El Soufi and Ilias [7] proved that it is always bounded among conformal metrics. Generalizing a result by Li and Yau [13] in dimension 2, they gave an explicit upper bound thanks to the $m$-conformal volume $V_{c}(m, M,[g])$ of $(M,[g])$

$$
\Lambda_{1}(M,[g]) \leq n V_{c}(m, M,[g])^{\frac{2}{n}}
$$


These conformal invariants on the standard sphere ( $\mathbb{S}^{n}$, [can]) satisfy (cf. [7])

$$
\Lambda_{1}\left(\mathbb{S}^{n},[\mathrm{can}]\right)=n \omega_{n}^{\frac{2}{n}}=n V_{c}\left(\mathbb{S}^{n},[\mathrm{can}]\right)^{\frac{2}{n}}
$$

and this value is achieved if and only if the metric is round. Here, $\omega_{n}$ denotes the volume of the standard $n$-sphere. Colbois and El Soufi [4] also proved that, for any compact Riemannian manifold $(M, g)$ of dimension $n \geq 3$,

$$
\Lambda_{1}(M,[g]) \geq \Lambda_{1}\left(\mathbb{S}^{n},[\mathrm{can}]\right) .
$$

We prove here that the case of equality characterizes the standard sphere :

Theorem 1. Let $(M, g)$ be a compact Riemannian manifold without boundary of dimension $n \geq 3$. Then

$$
\Lambda_{1}(M,[g])>\Lambda_{1}\left(\mathbb{S}^{n},[\mathrm{can}]\right)
$$

if $(M,[g])$ is not conformally diffeomorphic to $\left(\mathbb{S}^{n},[\mathrm{can}]\right)$.

This theorem answers the question raised in [2] and [11]. Note that a similar result was proved by the author in dimension 2 (see [14]). Note also that thanks to (1) and (2), the theorem implies

$$
V_{c}(m, M,[g])>\omega_{n}=V_{c}\left(\mathbb{S}^{n},[\mathrm{can}]\right)
$$

if $(M,[g])$ is not conformally diffeomorphic to ( $\mathbb{S}^{n}$, [can]). This gives a positive answer to Question 2 in [13].

In the rest of this paper, we prove the theorem. Based on the idea of Ledoux [12] and Druet [5], we start from a sharp Sobolev inequality in dimensions $n \geq 3$ (see $[9,5,6])$ which possesses extremal functions. These extremal functions give natural metrics $\tilde{g} \in[g]$ with

$$
\operatorname{Vol}_{\tilde{g}}(M)=1 \quad \text { and } \quad \lambda_{1}(\tilde{g}) \geq n \omega_{n}^{\frac{2}{n}} .
$$

As in dimension 2, see [14], we deal with the degeneracy consequences of the hypothesis $\lambda_{1}(\tilde{g})=n \omega_{n}^{\frac{2}{n}}$.

Let $(M, g)$ be a smooth compact Riemannian manifold of dimension $n \geq 3$ with $\operatorname{Vol}_{g}(M)=1$, which is not conformally diffeomorphic to the standard sphere. For an integer $m \geq 1$, let $h \in \mathrm{C}^{m}(M)$. We let $J_{g, h}$ be the functional defined for $u \in W^{1,2}(M) \backslash\{0\}$ by

$$
J_{g, h}(u)=\frac{\int_{M}|\nabla u|_{g}^{2} d v_{g}+\int_{M} h u^{2} d v_{g}-K_{n}^{-2}\left(\int_{M}|u|^{2^{*}} d v_{g}\right)^{\frac{2}{2^{*}}}}{\int_{M} u^{2} d v_{g}}
$$


where

$$
K_{n}=\frac{2}{\sqrt{n(n-2)}} \omega_{n}^{-\frac{1}{n}}
$$

is the sharp constant for the Sobolev inequality induced by the critical Sobolev embedding $W_{0}^{1,2} \subset L^{2^{*}}$ for bounded domains of $\mathbb{R}^{n}$, with $2^{*}=\frac{2 n}{n-2}$. Hebey and Vaugon proved in [9] that

$$
-\alpha(g, h)=\inf _{u \in W^{1,2}(M) \backslash\{0\}} J_{g, h}(u)
$$

is finite. Note that $J_{g, h}$ is scale invariant.

We will assume in the following that up to a conformal change, $g$ is a metric in $[g]$ with volume 1 which has a constant scalar curvature $S_{g}$. Since $M$ is not conformally diffeomorphic to the standard sphere, by the resolution of the Yamabe problem by Aubin [1] and Schoen [15], it satisfies

$$
\mu(M, g)<K_{n}^{-2}
$$

where $\mu(M, g)$ is the Yamabe invariant of $(M,[g])$. Let $V$ be an open neighbourhood of $\frac{n-2}{4(n-1)} S_{g}$ in $\mathrm{C}^{m}(M)$ such that

$$
\left\|h-\frac{n-2}{4(n-1)} S_{g}\right\|_{\infty} \leq \frac{1}{2}\left(K_{n}^{-2}-\mu(M, g)\right), \quad \text { for all } h \in V .
$$

Let $s \geq 0$ be such that $s+2>\frac{n}{2}$ and $m \geq s+2$. By the Sobolev embedding

$$
W^{s+2,2} \hookrightarrow \mathrm{C}^{0},
$$

the subset $W_{+}^{s+2,2}$ of positive functions of $W^{s+2,2}$ is open. We define

$$
\begin{aligned}
F: W_{+}^{s+2,2} \times \mathbb{R} \times V & \longrightarrow W^{s, 2}, \\
(u, \beta, h) & \longmapsto \Delta_{g} u+(h+\beta) u-K_{n}^{-2} u^{2^{*}-1},
\end{aligned}
$$

which is well defined because of the Sobolev algebra property of $W^{s+2,2}$ and $F$ is a $\mathcal{C}^{\infty}$ map. By a result of Druet [5], thanks to (6) and (7), for any $h \in V$, the functional $J_{g, h}$ attains its infimum. Let $u \in W^{1,2}(M)$ be such that

$$
J_{g, h}(u)=-\alpha(g, h) .
$$

Up to replace $u$ by $|u|$ and up to normalize, we can take

$$
u \geq 0 \text { and } \int_{M} u^{2^{*}} d v_{g}=1 .
$$


Then, $u$ satisfies the Euler-Lagrange equation

$$
F(u, \alpha(g, h), h)=\Delta_{g} u+(h+\alpha(g, h)) u-K_{n}^{-2} u^{2^{*}-1}=0
$$

where, by elliptic regularity theory, $u \in \mathfrak{e}^{m+2}$ and, by the maximum principle, $u>0$.

Let $v \in \mathcal{C}^{\infty}(M)$ and $t \in \mathbb{R}$ such that $|t|<\|v\|_{\infty}^{-1}$. Since $u$ is a minimum for (5),

$$
\begin{aligned}
& \int_{M}|\nabla(u+t u v)|_{g}^{2} d v_{g}+\int_{M}(h+\alpha(g, h))(u+t u v)^{2} d v_{g} \\
& -K_{n}^{-2}\left(\int_{M}(u+t u v)^{2^{\star}} d v_{g}\right)^{\frac{2}{2 \star}} \geq 0 .
\end{aligned}
$$

Since $u$ satisfies (8), the left term in (9) vanishes until the order 2 in the Taylor development as $t \rightarrow 0$. Computing the second-order coefficient as $t \rightarrow 0$, one gets

$$
\begin{aligned}
& \int_{M}|\nabla(u v)|_{g}^{2} d v_{g}+\int_{M}(h+\alpha(g, h))(u v)^{2} d v_{g} \\
& \quad-K_{n}^{-2}\left(2^{\star}-1\right) \int_{M} v^{2} u^{2^{\star}} d v_{g}+K_{n}^{-2}\left(2^{\star}-2\right)\left(\int_{M} v u^{2^{\star}} d v_{g}\right)^{2} \geq 0 .
\end{aligned}
$$

We now use the conformal transformation of the conformal Laplacian

$$
u^{2^{*}-1} \Delta_{\tilde{g}} v=\Delta_{g}(u v)-v \Delta_{g} u, \quad \text { for all } v \in \mathrm{C}^{\infty}(M),
$$

where

$$
\tilde{g}=u^{\frac{4}{n-2}} g .
$$

We integrate (11) against $u v$ and with (8),

$$
\begin{aligned}
& \int_{M}|\nabla(u v)|_{g}^{2} d v_{g} \\
& =\int_{M}|\nabla v|_{\tilde{g}}^{2} d v_{\tilde{g}}+\int_{M} v^{2} u \Delta_{g} u d v_{g} \\
& =\int_{M}|\nabla v|_{\tilde{g}} d v_{\tilde{g}}^{2}-\int_{M}(h+\alpha(g, h)) v^{2} u^{2} d v_{g}+K_{n}^{-2} \int_{M} v^{2} u^{2^{\star}} d v_{g}
\end{aligned}
$$

and with (4), (10) becomes

$$
\int_{M}|\nabla v|_{\tilde{g}}^{2} d v_{\tilde{g}}-n \omega_{n}^{\frac{2}{n}} \int_{M}\left(v-\int_{M} v d v_{\tilde{g}}\right)^{2} d v_{\tilde{g}} \geq 0 .
$$


This gives that $\lambda_{1}(\tilde{g}) \geq n \omega_{n}^{\frac{2}{n}}$. Note that if the inequality is strict for one solution $(h, u)$ of $F(u, \alpha(g, h), h)=0$, the theorem is proved.

We now assume that for any solution $(h, u)$ of $F(u, \alpha(g, h), h)=0$, we have $\lambda_{1}\left(u^{\frac{4}{n-2}} g\right)=n \omega_{n}^{\frac{2}{n}}$. We will apply the following theorem $([10]$, Theorem 5.4 , p. 63) of Fredholm theory to $F$, with $U=W_{+}^{s+2,2}(M) \times \mathbb{R}$.

Theorem 2. Let $X, Y$ be two separable Banach spaces, $U$ an open set of $X, V$ a separable $\mathrm{e}^{\infty}$ Banach manifold and $F \in \mathrm{e}^{\infty}(U \times V, Y)$ which satisfy:

- for all $(u, v) \in F^{-1}(0), D F(u)$ is surjective;

- for all $(u, v) \in F^{-1}(0), D_{u} F(u, v)$ is a Fredholm operator.

Then there exists a countable intersection of open dense sets (a residual set) $\Sigma \subset V$ such that for all $v \in \Sigma$, and for all $u \in F(., v)^{-1}(0), D_{u} F(u, v)$ is surjective.

Using (11) and (4), one gets for $(u, \beta, h) \in F^{-1}(0)$,

$$
D_{(u, \beta)} F(u, \beta, h) .(\theta, \mu)=u^{2^{*}-1}\left(\Delta_{\tilde{g}}\left(\frac{\theta}{u}\right)-n \omega_{n}^{\frac{2}{n}} \frac{\theta}{u}\right)+\mu u,
$$

where $\tilde{g}=u^{\frac{4}{n-2}} g$. Then, $D_{(u, \beta)} F(u, \beta, h)$ is a Fredholm operator. It remains to prove that if $(u, \beta, h) \in F^{-1}(0), D F(u, \beta, h)$ is surjective. We have

$$
D F(u, \beta, h) .(\theta, \mu, \tau)=u^{2^{*}-1}\left(\Delta_{\tilde{g}}\left(\frac{\theta}{u}\right)-n \omega_{n}^{\frac{2}{n}} \frac{\theta}{u}\right)+\mu u+\tau u .
$$

$\operatorname{Im}\left(D_{(u, \beta)} F(u, \beta, h)\right)$ is a closed space in $W^{s, 2}$ of finite codimension. Thus, since $\operatorname{Im}(D F(u, \beta, h))$ contains $\operatorname{Im}\left(D_{(u, \beta)} F(u, \beta, h)\right)$, it is a closed space in $W^{s, 2}$ by the following lemma.

Lemma. Let $X$ a Banach space, and $E \subset F \subset X$ some subspaces. If $E$ is a closed finite codimensional subspace of $X$, then $F$ is a closed subspace of $X$.

Proof. Let $G$ a finite dimensional subspace of $X$ such that $X=E \oplus G$. We set $H=G \cap F$. Then, $F=E \oplus H$. Let $x_{k} \in F$ such that $x_{k} \rightarrow x$ as $k \rightarrow+\infty$. We denote $x_{k}=y_{k}+z_{k}$ with $y_{k} \in E$ and $z_{k} \in H$.

We suppose that $\left(z_{k}\right)_{k \geq 0}$ is not bounded. Then, up to the extraction of a subsequence, $\left|z_{k}\right| \rightarrow+\infty$ as $k \rightarrow+\infty$. By Bolzano's theorem, up to the extraction of a subsequence, there exists $z \in H$ such that

$$
\frac{z_{k}}{\left|z_{k}\right|} \longrightarrow z, \quad \text { as } k \rightarrow+\infty
$$


Since $\left(x_{k}\right)$ converges as $k \rightarrow+\infty$,

$$
\frac{y_{k}}{\left|z_{k}\right|}=\frac{x_{k}}{\left|z_{k}\right|}-\frac{z_{k}}{\left|z_{k}\right|} \longrightarrow-z, \quad \text { as } k \rightarrow+\infty .
$$

Since $E$ is closed, we get $z \in E \cap H=0$, which contradicts $|z|=1$.

Then $\left(z_{k}\right)_{k \geq 0}$ is bounded and by Bolzano's theorem, up to the extraction of a subsequence, we can suppose that $z_{k} \rightarrow z \in H$ as $k \rightarrow+\infty$. Then,

$$
y_{k}=x_{k}-z_{k} \longrightarrow x-z, \quad \text { as } k \rightarrow+\infty .
$$

and $y=x-z \in E$ since $E$ is closed. Therefore $x=y+z \in E+H=F$ and the proof of the lemma is complete.

Now, it suffices to prove that $\operatorname{Im}(D F(u, \beta, h))^{\perp}=0$, where $\perp$ refers to the orthogonal in $W^{s, 2}$. Let $\phi \in \operatorname{Im}(D F(u, \beta, h))^{\perp}$. Then, with (14),

$$
\langle\phi, u \tau\rangle_{W^{s, 2}}=0, \quad \text { for all } \tau \in \mathcal{C}^{m} .
$$

Since $u \in \mathrm{e}^{m}$ is positive and $\mathrm{e}^{m}$ is dense in $W^{s, 2}$, we get $\phi=0$.

By Theorem 2, there exists $h \in V$ such that for all couple $(u, \beta)$ satisfying $F(u, \beta, h)=0, D F_{(u, \beta)}(u, \beta, h)$ is surjective. We take in particular $\beta=\alpha(g, h)$ and we will deduce that for a minimal function $u, \lambda_{1}(\tilde{g})=n \omega_{n}^{\frac{2}{n}}$ is simple with $\tilde{g}=u^{\frac{4}{n-2}} g$. We claim that

$$
\int_{M} u^{2} \phi d v_{g} \neq 0, \quad \text { for all } \phi \in E_{1}(\tilde{g}) \backslash\{0\} .
$$

Indeed, if $\phi$ is an eigenfunction for $\lambda_{1}(\tilde{g})$ such that this integral vanishes, one easily checks with (13) that $u \phi$ is orthogonal to the image of $D_{(u, \beta)} F(u, \alpha(h, g), h)$ in $L^{2}(g)$. It implies $\phi=0$ and we obtain (15). Since a bounded linear form vanishes on a one-codimensional space, we get that $\lambda_{1}(\tilde{g})$ is simple. Thus, $\lambda_{1}(\tilde{g})$ cannot be an extremal eigenvalue in the sense of [8] and as a result, $\lambda_{1}(\tilde{g})=n \omega_{n}^{\frac{2}{n}}$ is not locally maximal. The proof of Theorem 1 for $n \geq 3$ is complete.

Acknowledgements. I would like to thank my thesis advisor O. Druet for having pointed out to me the interest of Sobolev inequalities for the existence of metrics with large first eigenvalue and the referee for remarks on the manuscript which led to improvements in the presentation. 


\section{References}

[1] T. Aubin, Équations différentielles non linéaires et problème de Yamabe concernant la courbure scalaire. J. Math. Pures Appl. (9) 55 (1976), no. 3, 269-296. MR 0431287 Zbl 0336.53033

[2] B. Colbois, Spectre conforme et métriques extrémales. In Séminaire de Théorie Spectrale et Géométrie. Vol. 22. Année 2003-2004. Université de Grenoble I, Institut Fourier, Saint-Martin-d'Hères, 2004, 93-101. MR 2136138 MR 2136131 (collection) Zbl 1069.58017 Zbl 1054.35001 (collection)

[3] B. Colbois and J. Dodziuk, Riemannian metrics with large $\lambda_{1}$. Proc. Amer. Math. Soc. 122 (1994), no. 3, 905-906. MR 1213857 Zbl 0820.58056

[4] B. Colbois and A. El Soufi. Extremal eigenvalues of the Laplacian in a conformal class of metrics: the 'conformal spectrum'. Ann. Global Anal. Geom. 24 (2003), no. 4, 337-349. MR 2015867 Zbl 1036.58026

[5] O. Druet, Optimal Sobolev inequalities and extremal functions. The threedimensional case. Indiana Univ. Math. J. 51 (2002), no. 1, 69-88. MR 1896157 Zbl 1896157

[6] O. Druet and E. Hebey, The $A B$ program in geometric analysis: sharp Sobolev inequalities and related problems. Mem. Amer. Math. Soc. 160 (2002), no. 761. MR 1938183 Zbl 1023.58009

[7] A. El Soufi and S. Ilias, Immersions minimales, première valeur propre du laplacien et volume conforme. Math. Ann. 275 (1986), no. 2, 257-267. MR 0854009 Zbl 0675.53045

[8] A. El Soufi and S. Ilias, Laplacian eigenvalue functionals and metric deformations on compact manifolds. J. Geom. Phys. 58 (2008), no. 1, 89-104. MR 2378458 Zbl 1137.49040

[9] E. Hebey and M. Vaugon, Meilleures constantes dans le théorème d'inclusion de Sobolev et multiplicité pour les problèmes de Nirenberg et Yamabe. Indiana Univ. Math. J. 41 (1992), no. 2, 377-407. MR 1183349 Zbl 0764.53029

[10] D. Henry, Perturbation of the boundary in boundary-value problems of partial differential equations. With editorial assistance from J. Hale and A. L. Pereira. London Mathematical Society Lecture Note Series, 318. Cambridge University Press, Cambridge, 2005. MR 2160744 Zbl 1170.35300

[11] G. Kokarev and N. Nadirashvili, On first Neumann eigenvalue bounds for conformal metrics. In A.+ Laptev (ed.), Around the research of Vladimir Maz'ya. Vol. II. Partial differential equations. International Mathematical Series (New York), 12. Springer, Dordrecht etc., and Tamara Rozhkovskaya Publisher, Novosibirsk, 2010, 229-238. MR 2676176 MR 2664211 (collection) Zbl 1187.58030 Zbl 1180.47002 (collection)

[12] M. Ledoux, The geometry of Markov diffusion generators. Ann. Fac. Sci. Toulouse Math. (6) 9 (2000), no. 2, 305-366. Probability theory. MR 1813804 Zbl 0980.60097 
[13] P. Li and S. T. Yau, A new conformal invariant and its applications to the Willmore conjecture and the first eigenvalue of compact surfaces. Invent. Math. 69 (1982), no. 2, 269-291. MR 0674407 Zbl 0503.53042

[14] R. Petrides, Existence and regularity of maximal metrics for the first Laplace eigenvalue on surfaces. Geom. Funct. Anal. 24 (2014), no. 4, 1336-1376. MR 3248488 Zbl 06381497

[15] R. Schoen, Conformal deformation of a Riemannian metric to constant scalar curvature. J. Differential Geom. 20 (1984), no. 2, 479-495. MR 0788292 Zbl 0576.53028

[16] P. C. Yang and S. T. Yau, Eigenvalues of the Laplacian of compact Riemann surfaces and minimal submanifolds. Ann. Scuola Norm. Sup. Pisa Cl. Sci. (4) 7 (1980), no. 1, 55-63. MR 0577325 Zbl 0446.58017

Received October 14, 2013; revised February 17, 2014

Romain Petrides, Université de Lyon, CNRS UMR 5208, Université Lyon 1, Institut Camille Jordan, 43 bd du 11 novembre 1918, F-69622 Villeurbanne cedex, France

e-mail: romain.petrides@univ-lyonl.fr 